\title{
The number of solutions of the Mordell equation
}

\author{
by \\ Dimitrios Poulakis (Thessaloniki) \\ To the memory of André Néron
}

1. Introduction. Let $a, b$ be integers such that the polynomial $f(x)=$ $x^{3}+a x+b$ has discriminant $\Delta(f) \neq 0$. In [3] Evertse and Silverman proved that the number $Z(f)$ of integer solutions of the equation $y^{2}=f(x)$ satisfies

$$
Z(f) \leq 7^{[L: \mathbb{Q}](4+9 s)} h_{2}(L)^{2}+3,
$$

where $s$ is the cardinality of the set containing the usual absolute value of $\mathbb{Q}$ and the $p$-adic absolute values $|\cdot|_{p}$ for which $|\Delta(f)|_{p} \neq 1, L$ the splitting field of $f(x)$ and $h_{2}(L)$ the order of the subgroup of the ideal class group of $L$ consisting of the ideal classes $[A]$ with $[A]^{2}=1$. Using this result Schmidt [7] proved that given $\varepsilon>0$ there is a constant $c(\varepsilon)$ depending on $\varepsilon$ such that

$$
Z(f) \leq c(\varepsilon)|\Delta(f)|^{1 / 2+\varepsilon} .
$$

In the case of the Mordell equation (i.e. $a=0$ ), it follows that $Z(f) \leq$ $c(\varepsilon)|b|^{1+\varepsilon}$. Moreover, Schmidt conjectured that the number of solutions $x, y \in \mathbb{Z}$ of an irreducible equation $F(x, y)=0$ defining a curve of positive genus having coefficients in $\mathbb{Z}$ and total degree $N$ is at most

$$
c(N, \varepsilon) H(F)^{\varepsilon},
$$

where $c(N, \varepsilon)$ is a constant depending on $N$ and $\varepsilon$.

In this paper we improve on the estimate of Schmidt for the Mordell equations by showing that the number of integer solutions of $y^{2}=x^{3}+$ $b$ depends only on the prime divisors of $b$. More precisely, we prove the following result:

Theorem 1. Let $k$ be a nonzero rational integer. Denote by $\omega(k)$ the number of prime divisors of $k$ and by $P(k)$ the product of all the prime divisors $p$ of $k$ with $p>3$. If $k$ has no prime divisors $>3$, put $P(k)=1$.

1991 Mathematics Subject Classification: 11D25, 11G05. 
Then the number of solutions $(x, y) \in \mathbb{Z}^{2}$ of the equation $y^{2}=x^{3}+k$ is

$$
<10^{11 \omega(k)+48} P(k) .
$$

COROLlary 1. Let $k$ be a nonzero rational integer and $\Pi(k)$ be the product of the prime divisors of $k$. Then for every $\varepsilon>0$ there is a constant $\Omega(\varepsilon)$, independent of $k$, such that the number of solutions $(x, y) \in \mathbb{Z}^{2}$ of the equation $y^{2}=x^{3}+k$ is

$$
<\Omega(\varepsilon) \Pi(k)^{1+\varepsilon} .
$$

The above theorem is a consequence of the following effective version of Shafarevich's theorem ([5, p. 222], [8, p. 263]):

Theorem 2. Let $S$ be a finite set of rational primes with $2,3 \in S$. Denote by $P(S)$ the product of all the primes $p$ in $S$ with $p>3$. If $S=\{2,3\}$, put $P(S)=1$. Then the number of $\mathbb{Q}$-isomorphism classes of elliptic curves over $\mathbb{Q}$ with good reduction outside $S$ is

$$
<10^{11 \sharp S+26} P(S) .
$$

In [1] there is an effective proof of Shafarevich's theorem using the estimate of [3]. Our approach is completely different and has the advantage that does not use the results of [3]. The only Diophantine approximation result we use is the estimate for the number of solutions of the $S$-units equation $x+y=1$ due to Evertse [2].

2. Auxiliary results. In this section we give some lemmas which will be useful for the proof of our results.

Lemma 1. Let $S$ be a finite set of rational primes with $2 \in S$ and $f(x)=$ $x^{3}+A x+B$ be a polynomial of $\mathbb{Z}[x]$ with distinct roots. Suppose that the elliptic curve $E: y^{2}=f(x)$ has good reduction outside $S$. Let $L=\mathbb{Q}(\theta)$, where $\theta$ is a root of $f(x)$. Suppose that $L \neq \mathbb{Q}$. Then the discriminant $D_{L}$ of $L$ has the form

$$
D_{L}= \pm 2^{\alpha} 3^{\beta} \prod_{p} p^{s_{p}},
$$

where the product is taken over all the primes $p \geq 5$, with $s_{p}=0$ for $p$ outside $S$ and $0 \leq s_{p} \leq \operatorname{deg} L-1$ for $p \in S$. Moreover, $\alpha=0,2,3$ and $\beta \leq 1$ if $\operatorname{deg} L=2$, while $\beta=0,1,3,4,5$ if $\operatorname{deg} L=3$.

Proof. The nonzero points of 2-torsion of $E$ are the points $\left(0, \theta_{i}\right)(i=$ $1,2,3)$ where $\theta_{1}, \theta_{2}, \theta_{3}$ are the roots of $f(x)$. By [5, Theorem 1, p. 113], the extension $\mathbb{Q}\left(\theta_{1}, \theta_{2}, \theta_{3}\right) / \mathbb{Q}$ is unramified outside $S$. Then the extension $L / \mathbb{Q}$ is unramified outside $S$, whence the prime divisors of $D_{L}$ are primes in $S$. Hence,

$$
D_{L}= \pm 2^{\alpha} 3^{\beta} \prod_{p} p^{s_{p}},
$$


where the product is taken over all the primes $p \geq 5$, with $s_{p}=0$ for $p$ outside $S$. If $L$ is a quadratic extension, then $\alpha=0,2$ or $3, \beta \leq 1$ and $s_{p} \leq 1$ for $p \in S$. If $L$ is a cubic extension, [6, Theorem 2] implies that $\alpha=0,2$ or $3, \beta=0,1,3,4$ or 5 and $s_{p} \leq 2$ for $p \in S$.

Lemma 2. Let $D$ be an integer. Then the number of cubic fields of discriminant $D$ is at most $546|D|^{1 / 2}$.

Pr o of. Let $K$ be a cubic field of discriminant $D$. Then [4, pp. 620-625] implies that $|D| \geq 23$. Let $\sigma_{1}, \sigma_{2}, \sigma_{3}$ be the embeddings of $K$ into the field $\mathbb{C}$ of complex numbers. We denote by $s$ and $2 t$ the number of real and complex embeddings respectively. If $s=t=1$, let $\sigma_{2}, \sigma_{3}$ be the complex embeddings. As usual denote complex conjugation by bars and define $\bar{\sigma}_{i}(x)=\overline{\sigma_{i}(x)}$. Thus $\sigma_{3}=\bar{\sigma}_{2}$. The map $\sigma: K \rightarrow \mathbb{R}^{s} \times \mathbb{C}^{t}$ given by $\sigma(x)=\left(\sigma_{1}(x), \ldots, \sigma_{3-t}(x)\right)$ defines an embedding of $K$ into $\mathbb{R}^{s} \times \mathbb{C}^{t}$. The image $\sigma\left(O_{K}\right)$ of the ring $O_{K}$ of algebraic integers of $K$ is a lattice in $\mathbb{R}^{s} \times \mathbb{C}^{t}$. In [4, Chapter 28, $\S 1]$ a structure of Euclidean space is defined on $\mathbb{R}^{s} \times \mathbb{C}^{t}$. The fundamental parallelotope of the lattice $\sigma\left(O_{K}\right)$ has content $|D|^{1 / 2}$ with respect to this Euclidean metric [4, p. 538].

Let $A$ be the convex region in $\mathbb{R}^{s} \times \mathbb{C}^{t}$ determined by the inequalities

$$
|x|+|y|+|z| \leq \varrho, \quad|x+y+z| \leq \varrho^{\prime}<\varrho \quad \text { if }(s, t)=(3,0)
$$

and

$$
|x|+|y|+|\bar{y}| \leq \varrho, \quad|x+y+\bar{y}| \leq \varrho^{\prime}<\varrho \quad \text { if }(s, t)=(1,1) .
$$

By $[4$, p. 623], the content of the region $A$ is

$$
\geq \frac{4}{3}\left(\frac{\pi}{4}\right)^{t} \varrho^{\prime} \varrho^{2}
$$

We choose $\varrho$ so that

$$
\frac{4}{3}\left(\frac{\pi}{4}\right)^{t} \varrho^{\prime} \varrho^{2} \geq 8|D|^{1 / 2}
$$

Putting $\varrho^{\prime}=\varrho / 2$, we can take $\varrho=(4 / \pi)^{t / 3} 12^{1 / 3}|D|^{1 / 6}$. Hence, Minkowski's lattice point theorem [5, p. 601] implies that there exists an algebraic integer $\xi$ of $K$ satisfying

$\left|\xi_{1}\right|+\left|\xi_{2}\right|+\left|\xi_{3}\right| \leq\left(\frac{4}{\pi}\right)^{t / 3} 12^{1 / 3}|D|^{1 / 6}, \quad\left|\xi_{1}+\xi_{2}+\xi_{3}\right| \leq \frac{1}{2}\left(\frac{4}{\pi}\right)^{t / 3} 12^{1 / 3}|D|^{1 / 6}$,

where $\xi_{1}, \xi_{2}, \xi_{3}$ are the conjugates of $\xi$.

The arithmetic-geometric inequality implies

$$
\left|\xi_{1} \xi_{2} \xi_{3}\right|<|D|^{1 / 2}
$$

For arbitrary real numbers $a, b, c$ we have the inequality

$$
a b+b c+a c \leq \frac{1}{2}(a+b+c)^{2} .
$$


Hence

$$
\left|\xi_{1} \xi_{2}+\xi_{2} \xi_{3}+\xi_{1} \xi_{3}\right|<2|D|^{1 / 3} .
$$

Let $f(x)=x^{3}+A x^{2}+B x+C$ be the irreducible polynomial of $\xi$. Then

$$
|A|<2|D|^{1 / 6}, \quad|B|<2|D|^{1 / 3}, \quad|C|<|D|^{1 / 2} .
$$

The discriminant of $f(x)$ is

$$
\Delta=-4 A^{3} C+A^{2} B^{2}+18 A B C-4 B^{3}-27 C^{2} .
$$

Thus, the inequalities for $A, B, C$ give $|\Delta|<179|D|$. We denote by $i(\xi)$ the index of $\xi$. We have $\Delta=i(\xi)^{2} D$, whence $|i(\xi)| \leq 13$.

We now consider the surface given by the equation

$$
F(X, Y, Z)=-4 X^{3} Z+X^{2} Y^{2}+18 X Y Z-4 Y^{3}-27 Z^{2}-D L^{2}=0,
$$

where $L$ is a positive integer with $L \leq 13$. The number of triples $(u, v, w) \in$ $\mathbb{Z}^{3}$ with $|u|<2|D|^{1 / 6},|v|<2|D|^{1 / 3}$ and $|w|<|D|^{1 / 2}$ satisfying $F(u, v, w)=$ 0 is less than $2\left(4|D|^{1 / 6}+1\right)\left(4|D|^{1 / 3}+1\right)<42|D|^{1 / 2}$ (we have used the fact that $|D| \geq 23)$. Since we have at most 13 choices for $L$, the lemma follows.

LEMma 3. Let $K$ be an algebraic number field of degree $d$ and $S$ be a finite set of places on $K$ containing all the infinite places of $K$. Then the equation $x+y=1$ has at most

$$
3 \cdot 7^{d+2 \sharp S}
$$

solutions in $S$-units $x, y$ of $K$.

Proof. See [2].

LEMMA 4. Let $K$ be an algebraic number field and $L$ be a Galois extension of $K$ of degree $l$. Then each $L$-isomorphism class of elliptic curves defined over $K$ splits into at most $6^{l} K$-isomorphism classes.

Proof. Let $E$ and $A$ be two elliptic curves defined over $K$ and let $\alpha$ : $E \rightarrow A$ be an isomorphism over $L$. Then we have a map $F(\alpha): \operatorname{Gal}(L / K) \rightarrow$ $\operatorname{Aut}(E)$ defined by

$$
F(\alpha)(\sigma)=\alpha^{-1} \circ \alpha^{\sigma} \quad \text { for every } \sigma \in \operatorname{Gal}(L / K) .
$$

Suppose now that $B$ is another elliptic curve defined over $K$ and $\beta: E \rightarrow B$ an $L$-isomorphism with $F(\alpha)=F(\beta)$. It follows that

$$
\alpha^{-1} \circ \alpha^{\sigma}=\beta^{-1} \circ \beta^{\sigma} \quad \text { for every } \sigma \in \operatorname{Gal}(L / K) \text {. }
$$

Setting $\lambda=\beta \circ \alpha^{-1}$, we have $\lambda^{\sigma}=\lambda$ for every $\sigma \in \operatorname{Gal}(L / K)$. So, the isomorphism $\lambda$ is defined over $K$, whence $A$ and $B$ are $K$-isomorphic. Thus, given an $L$-isomorphism class $C$ of elliptic curves defined over $K$, the map $\alpha \rightarrow F(\alpha)$ defines an injection from the set of pairwise distinct $K$-isomorphism classes belonging to $C$ into the set of maps from $\operatorname{Gal}(L / K)$ 
to $\operatorname{Aut}(E)$. Since the cardinality of $\operatorname{Gal}(L / K)$ is $l$ and that of $\operatorname{Aut}(E)$ is at most 6 , the lemma follows.

3. Proof of Theorem 2. Let $E: y^{2}=x^{3}+A x+B$, where $A, B \in \mathbb{Z}$, be an elliptic curve having good reduction outside $S$. We denote by $L$ the field obtained by adjoining to $\mathbb{Q}$ the points of order 2 of $E$. It is the field generated over $\mathbb{Q}$ by the roots of $x^{3}+A x+B$. We have the following cases.

1. $L=\mathbb{Q}$. Then $E$ is isomorphic over $\mathbb{Q}$ to an elliptic curve in Legendre form

$$
E_{\lambda}: \quad y^{2}=x(x-1)(x-\lambda)
$$

where $\lambda \in \mathbb{Q}$. The $j$-invariant of $E_{\lambda}$ is

$$
j=2^{8} \frac{\left(\lambda^{2}-\lambda+1\right)^{3}}{\lambda^{2}(\lambda-1)^{2}} .
$$

Since $E$ has good reduction outside $S, j$ is a $S$-integer of $\mathbb{Q}$. Let $|\cdot|_{p}$ be a $p$-adic absolute value with $p$ outside $S$. If $|\lambda|_{p} \neq 1$, then $|j|_{p}>1$ from the equation for $j$, contradicting the fact that $j$ is a $S$-integer. It follows that $\lambda$ is a $S$-unit. Similarly for $1-\lambda$. Thus, $\lambda$ and $\mu=1-\lambda$ are two $S$-units satisfying $\lambda+\mu=1$. By Lemma 3 , the number of $S$-units $x, y$ of $\mathbb{Q}$ with $x+y=1$ is at most $3 \cdot 7^{3+2 \sharp S}$, whence there are at most $3 \cdot 7^{3+2 \sharp S}$ choices for $\lambda$. Hence, there are at most $3 \cdot 7^{3+2 \sharp S} \mathbb{Q}$-isomorphism classes of elliptic curves $E$ over $\mathbb{Q}$ with good reduction outside $S$ such that the points of order 2 of $E$ are defined over $\mathbb{Q}$.

2. $[L: \mathbb{Q}]=2$. Let $\Sigma$ be the set of prime ideals of $L$ lying above the elements of $S$. The curve $E$ is isomorphic over $L$ to an elliptic curve in Legendre form

$$
E_{\lambda}: \quad y^{2}=x(x-1)(x-\lambda),
$$

where $\lambda \in L$. Then we deduce as in case 1 that there are at most $3 \cdot 7^{4+2 \sharp \Sigma}$ choices for $\lambda$. Hence, there are at most $3 \cdot 7^{4+4 \sharp S} L$-isomorphism classes of elliptic curves $E$ over $\mathbb{Q}$ with good reduction outside $S$. Let $L=\mathbb{Q}(\sqrt{d})$, where $d$ is a squarefree rational integer. Then the discriminant $D_{L}$ of $L$ is $d$ or $4 d$. On the other hand, Lemma 1 yields

$$
D_{L}= \pm 2^{\alpha} 3^{\beta} \prod_{p} p^{s_{p}}
$$

where the product is taken over all the primes $p \geq 5$, with $s_{p}=0$ for $p$ outside $S, 0 \leq s_{p} \leq 1$ for $p \in S$ and $\alpha \leq 3, \beta \leq 1$. It follows that there exist $2^{4+\sharp S}$ choices for $L$. Furthermore, Lemma 4 implies that every $L$-isomorphism class of elliptic curves over $\mathbb{Q}$ is divided into at most 36 pairwise distinct $\mathbb{Q}$-isomorphism classes of elliptic curves over $\mathbb{Q}$. Thus, we 
conclude that there are less than

$$
108 \cdot 7^{4+4 \sharp S} \cdot 2^{4+\sharp S}
$$

$\mathbb{Q}$-isomorphism classes of elliptic curves $E$ over $\mathbb{Q}$ with good reduction outside $S$ with exactly one nonzero point of order 2 defined over $\mathbb{Q}$.

3. $[L: \mathbb{Q}]=3$ or 6 . Let $K=\mathbb{Q}(\theta)$, where $\theta$ is a root of the polynomial $x^{3}+A x+B$. By Lemma 1 , the discriminant of $K$ is

$$
D_{K}= \pm 2^{\alpha} 3^{\beta} \prod_{p} p^{s_{p}},
$$

where $\alpha=0,2$ or $3, \beta=0,1,3,4$ or 5 and the product is over all primes $p \geq$ 5 , with $s_{p}=0$ for $p$ outside $S$ and $0 \leq s_{p} \leq 2$ for $p \in S$. If $S \neq\{2,3\}$, then we denote by $P(S)$ the product of the primes of $S-\{2,3\}$ and if $S=\{2,3\}$, we put $P(S)=1$. By Lemma 2 , there are at most $24570 P(S)$ cubic fields of given discriminant $D_{K}$. On the other hand, there are at most $10 \cdot 3^{\sharp S-1}$ choices for $D_{K}$. Hence, the number of choices for $K$ and therefore for $L$ is

$$
<81900 \cdot 3^{\sharp S} P(S) .
$$

If $[L: \mathbb{Q}]=3$, we conclude, as in the previous cases, that there are less than $3 \cdot 7^{9+6 \sharp S}$ choices for the $L$-isomorphism class of $E$ and Lemma 4 implies that every such class splits into $6^{3} L$-isomorphism classes of elliptic curves over $\mathbb{Q}$. It follows that the number of $\mathbb{Q}$-isomorphism classes of elliptic curves $E$ over $\mathbb{Q}$ with good reduction outside $S$ such that their 2-torsion points generate over $\mathbb{Q}$ a cubic extension is

$$
<3 \cdot 10^{15} \cdot 3^{\sharp S} \cdot 7^{6 \sharp S} P(S) .
$$

If $[L: \mathbb{Q}]=6$, we deduce that there are less than $3 \cdot 7^{18+12 \sharp S}$ choices for the $L$-isomorphism class of $E$ and Lemma 4 yields that every such class splits into $6^{6} L$-isomorphism classes of elliptic curves over $\mathbb{Q}$. Thus, the number of $\mathbb{Q}$-isomorphism classes of elliptic curves $E$ over $\mathbb{Q}$ with good reduction outside $S$ such that their 2-torsion points generates over $\mathbb{Q}$ an extension of degree 6 is

$$
<2 \cdot 10^{25} \cdot 3^{\sharp S} \cdot 7^{12 \sharp S} P(S) .
$$

Summarizing our estimates, we deduce that the number of $\mathbb{Q}$-isomorphism classes of elliptic curves $E$ over $\mathbb{Q}$ with good reduction outside $S$ is

$$
<10^{11 \sharp S+26} P(S) \text {. }
$$

4. Proof of Theorem 1. We shall follow the idea of [8, Remark 6.5, p. 265]. Let $(u, v) \in \mathbb{Z}^{2}$ be a solution of the Mordell equation $y^{2}=x^{3}+k$. We associate with this solution the elliptic curve $E(u, v)$ defined by the equation

$$
Y^{2}=X^{3}-3 u X+2 v .
$$


The discriminant of $E(u, v)$ is

$$
16\left(4(3 u)^{3}-27(2 v)^{2}\right)=-2^{6} 3^{3} k .
$$

It follows that $E(u, v)$ has good reduction outside 2,3 and the primes dividing $k$. Suppose now that $(w, z) \in \mathbb{Z}^{2}$ is another solution such that the curves $E(w, z)$ and $E(u, v)$ are isomorphic over $\mathbb{Q}$. Then there is $a \in \mathbb{Q}$ such that $u=a^{4} w$ and $v=a^{6} z$, whence we get

$$
k=v^{2}-u^{3}=a^{12}\left(y^{2}-x^{3}\right)=a^{12} k .
$$

Since $a \in \mathbb{Q}$, we obtain $a= \pm 1$. So $(u, v)=(w, z)$. Hence, distinct solutions $(u, v)$ of the Mordell equation correspond to distinct $\mathbb{Q}$-isomorphism classes of elliptic curves with good reduction outside 2, 3 and the primes dividing $k$. Let $\omega(k)$ be the number of prime divisors of $k$ and $P(k)$ be the product of the prime divisors $p$ of $k$ with $p>3$. If the divisors of $k$ are among 2 and 3 , we put $P(k)=1$. Thus, Theorem 2 implies that the number of solutions $(x, y) \in \mathbb{Z}^{2}$ to the equation $y^{2}=x^{3}+k$ is $<10^{11 \omega(k)+48} P(k)$.

Acknowledgements. The author wishes to thank the referee for several helpful suggestions and comments.

\section{References}

[1] A. Brumer and J. Silverman, The number of elliptic curves over $\mathbb{Q}$ with conductor $N$, Manuscripta Math. 91 (1996), 95-102.

[2] J. H. Evertse, On equations in S-units and the Thue-Mahler equation, Invent. Math. 75 (1984), 561-584.

[3] J. H. Evertse and J. H. Silverman, Uniform bounds for the number of solutions to $Y^{m}=f(X)$, Math. Proc. Cambridge Philos. Soc. 100 (1986), 237-248.

[4] H. Hasse, Number Theory, Springer, Berlin, 1980.

[5] S. Lang, Elliptic Functions, Addison-Wesley, 1973.

[6] P. Llorente and E. Nart, Effective determination of the decomposition of the rational primes in a cubic field, Proc. Amer. Math. Soc. 87 (1983), 579-585.

[7] W. M. Schmidt, Integer points on curves of genus 1, Compositio Math. 81 (1992), $33-59$.

[8] J. H. Silverman, The Arithmetic of Elliptic Curves, Grad. Texts in Math. 106, Springer, New York, 1986.

Department of Mathematics Aristotle University of Thessaloniki

54006 Thessaloniki, Greece

E-mail: poulakis@ccf.auth.gr 